Multiples Myelom

\title{
Dauerhaft den Tumor in Schach halten
}

\section{Bei Patienten mit einem Multiplen Myelom (MM) erniedrigt die kon- tinuierliche Einnahme von Lenalidomid (Revlemid ${ }^{\oplus}$ ) nach erfolgter Hochdosis-Chemotherapie mit autologer Stammzelltransplantation das Risiko für Krankheitsprogression und Tod um $56 \%$ gegenüber Placebo, erklärte Kenneth C. Anderson, Los Angeles.}

\begin{abstract}
Aktualisierten Daten der klinischen CALGB-100104-Studie zufolge erspare man den Patienten mit der Erhaltungstherpie im Vergleich zu Standardtherapien Blutbildveränderungen, Osteolysen oder Organschädigungen. Lenalidomid wirke sowohl auf die Tumorzellen selbst als auch die Mikroumgebung im Knochenmark, was die Antitumor-Aktivität verstärke, so Anderson. Als Beispiel für eine erfolgreiche Erhaltungstherapie mit Lenalidomid stellte
\end{abstract}

Anderson einen Patienten vor, der seit über zehn Jahren unter einem MM leidet. Nach mehreren Vorbehandlungen und Krankheitsrückfällen erhielt er als einer der Ersten vor etwa neun Jahren Lenalidomid als orale Dauertherapie. Seither ist der Patient weitgehend beschwerdefrei und führt ein normales Privat- und Berufsleben, betonte Anderson.

Auch ältere Patienten, für die eine Stammzelltransplantation nicht mehr in Frage kommt, pro- fitierten von der kontinuierlichen Lenalidomid-Therapie, ergänzte Mario Boccadoro, Turin. Er skizzierte die Daten einer internationalen multizentrischen Phase-IIIStudie mit 459 MM-Patienten. In dieser Studie wurde in drei Arme randomisiert - MPR-R: Melphalan, Prednison, Lenalidomid plus nachfolgender LenalidomidDauertherapie, MPR: Melphalan, Prednison, Lenalidomid und MP: Melphalan, Prednison. Nach einem mittleren Nachbeobachtungszeitraum von 25 Monaten ergab sich für die Patienten, die eine zusätzliche LenalidomidDauertherapie erhalten hatten insgesamt ein um $60 \%$ reduziertes Progressionsrisiko. zi

Mediaworkshop im Rahmen des XIII. International Myeloma Workshop am 5. Mai 2011 in Paris/Frankreich; Veranstalter: Celgene
Bendamustin auf dem Vormarsch? Löst Bendamustin andere Chemotherapien bei Follikulären Lymphomen und CLL ab? Anlässlich des Europäischen Hämatologenkongresses (EHA) im Juni 2011 in London wurde die Frage aufgeworfen, welches die optimale Induktionstherapie für Follikuläre Lymphome sei. Nach Meinung von Wolfram Brugger, Villingen-Schwenningen, ist die Kombination aus Bendamustin (Ribomus$\operatorname{tin}^{\oplus}$ ) und Rituximab (B-R) der neue Maßstab und gehöre in Deutschland bereits zum empfohlenen Therapiestandard für ältere Patienten. In den USA ist B-R seit diesem Jahr in den NCCN (National Comprehensive Cancer Network)-Empfehlungen zur Erstlinientherapie etabliert (www.ncen.org/ professionals/physician_gls/f_guidelines. asp). „In Deutschland hat sich B-R bereits im Behandlungsalltag etabliert", betonte Brugger. Dies zeigen Daten aus dem deutschen Tumorregister Lymphatische Neoplasien, in dem B-R die am häufigsten durchgeführte Erstlinientherapie bei indolenten Lymphomen in der onkologischen Praxis repräsentiert.

Nach Informationen von Mundipharma
Everolimus be im fortgeschrittenen Nierenzellkarzinom

Der mTOR-Hemmer Everolimus (Afinitor ${ }^{\circledast}$ ) ist seit 2009 zur Behandlung von Patienten mit fortgeschrittenem Nierenzellkarzinom (mRCC) zugelassen, bei denen es während oder nach einer gegen den vaskulären Wachstumsfaktor (VEGF) gerichteten Therapie (z.B. Sunitinib, Pazopanib oder Sorafenib) zu einer Krankheitsprogression kommt. In der zulassungsrelevanten Phase-III-Studie RECORD (Renal Cell cancer treatment with Oral RAD001 given Daily)-1 lebten die mRCC-Patienten im Median 4,9 Monate progressionsfrei, wenn sie nach der Ersttherapie Everolimus erhalten hatten $(p<0,001)$ [Motzer RJ et al. Cancer. 2010;116(18):425665].

In der Zwischenanalyse der prospektiven, nicht interventionellen Studie CHANGE (CHarakterisierung von Afinitor Nach Gezielter Ersttherapie) ließ sich nun in dieser Situation eine mediane progressionsfreie Zeit bis zum Progress von 9,7 Monaten beobachten [Bergmann L et al. J Clin Oncol. 2011; 29 (suppl; abstr 4552)]. Im Praxisalltag ist die Zeit bis zum Krankheitsprogress damit noch deutlich länger als in der Zulassungsstudie.

Nach Informationen von Novartis Pharma

\section{Tumor-assoziierter Eisenmangel -} Erfolge mit Eisencarboxymaltose Die Ursache Tumor-bedingter Anämien ist in vielen Fällen ein funktioneller Eisenmangel, der mit einer Störung der intestinalen Eisenresorption und einer reduzierten Mobilisierung des Eisens aus den Speichern einhergeht. Eine Transferrinsättigung (TSAT) von $<20 \%$ ist wichtiger diagnostischer Marker für einen funktionellen Eisenmangel. Nach Tilman Steinmetz, Köln, zeitigt eine orale Eisentherapie bei funktionellem Eisenmangel so gut wie keine Wirkung. Dass mit einer intravenösen Gabe von Eisencarboxymaltose (Ferinject ${ }^{\oplus}$ ) gute Erfolge erzielt werden, belegte eine 12-wöchtige Beobachtungsstudie. Durchschnittlich benötigten die Patienten 1.333 mg Eisen, um den Hämoglobin ( $\mathrm{Hb}$ )-Wert zu normalisieren. Der größte Benefit wurde bei Patienten mit einem $\mathrm{Hb}$ von unter $10 \mathrm{~g} / \mathrm{dl}$ erreicht. Die Mehrzahl der Ärzte beurteilte Wirksamkeit und Verträglichkeit von Eisencarboxymaltose als gut und sehr gut. 